\title{
Lateral boundary contributions to wave- activity invariants and nonlinear stability theorems for balanced dynamics
}

Article

Published Version

Ren, S. and Shepherd, T. G. (1997) Lateral boundary contributions to wave-activity invariants and nonlinear stability theorems for balanced dynamics. Journal Of Fluid Mechanics, 345. pp. 287-305. ISSN 0022-1120 doi:

https://doi.org/10.1017/S0022112097006216 Available at https://centaur.reading.ac.uk/32862/

It is advisable to refer to the publisher's version if you intend to cite from the work. See Guidance on citing.

Published version at: http://dx.doi.org/10.1017/S0022112097006216

To link to this article DOI: http://dx.doi.org/10.1017/S0022112097006216

Publisher: Cambridge University Press

All outputs in CentAUR are protected by Intellectual Property Rights law, including copyright law. Copyright and IPR is retained by the creators or other copyright holders. Terms and conditions for use of this material are defined in the End User Agreement.

www.reading.ac.uk/centaur 
Central Archive at the University of Reading

Reading's research outputs online 


\title{
Lateral boundary contributions to wave-activity invariants and nonlinear stability theorems for balanced dynamics
}

\author{
By SHUZHAN REN AND THEODORE G. SHEPHERD \\ Department of Physics, University of Toronto, Toronto M5S 1A7 Canada
}

(Received 1 October 1996 and in revised form 15 April 1997)

The slow advective-timescale dynamics of the atmosphere and oceans is referred to as balanced dynamics. An extensive body of theory for disturbances to basic flows exists for the quasi-geostrophic (QG) model of balanced dynamics, based on wave-activity invariants and nonlinear stability theorems associated with exact symmetry-based conservation laws. In attempting to extend this theory to the semigeostrophic (SG) model of balanced dynamics, Kushner \& Shepherd discovered lateral boundary contributions to the SG wave-activity invariants which are not present in the QG theory, and which affect the stability theorems. However, because of technical difficulties associated with the SG model, the analysis of Kushner \& Shepherd was not fully nonlinear.

This paper examines the issue of lateral boundary contributions to wave-activity invariants for balanced dynamics in the context of Salmon's nearly geostrophic model of rotating shallow-water flow. Salmon's model has certain similarities with the SG model, but also has important differences that allow the present analysis to be carried to finite amplitude. In the process, the way in which constraints produce boundary contributions to wave-activity invariants, and additional conditions in the associated stability theorems, is clarified. It is shown that Salmon's model possesses two kinds of stability theorems: an analogue of Ripa's small-amplitude stability theorem for shallow-water flow, and a finite-amplitude analogue of Kushner \& Shepherd's SG stability theorem in which the 'subsonic' condition of Ripa's theorem is replaced by a condition that the flow be cyclonic along lateral boundaries. As with the SG theorem, this last condition has a simple physical interpretation involving the coastal Kelvin waves that exist in both models.

Salmon's model has recently emerged as an important prototype for constrained Hamiltonian balanced models. The extent to which the present analysis applies to this general class of models is discussed.

\section{Introduction}

In studying the dynamics of atmospheric and oceanic flows, theorists have found it useful to consider reduced models of the governing equations that filter fast degrees of freedom such as acoustic and inertia-gravity waves, leaving only the slow advectivetimescale dynamics associated with nonlinear vortical motion and Rossby waves. Such reduced models, frequently referred to as 'balanced' models, are more amenable to theoretical analysis than are the underlying 'primitive' equations. This notion of 
dynamical reduction onto a slow manifold can be formalized in terms of a slaving ansatz (e.g. Warn et al. 1995).

When a dynamical regime is characterizable in terms of certain dimensionless parameters, it may be possible to obtain a balanced model as the leading-order system in an asymptotic perturbation expansion in one or more small parameters. For example, one may obtain in this way quasi-geostrophic (QG) dynamics when the Rossby and Froude numbers are small and the characteristic lengthscale $L$ is sub-planetary, planetary geostrophic dynamics when the Rossby number is small and $L$ is much greater than the Rossby deformation radius, and two-dimensional semigeostrophic dynamics when the horizontal flow is anisotropic (see e.g. Pedlosky 1987). The last regime includes the special case of low-frequency coastally trapped waves.

Each of the three dynamical models mentioned above captures a range of important physical phenomena, and together they provide the basis for much of our theoretical understanding of large-scale atmospheric and oceanic flows. However, the assumption made in each case of a single well-defined parameter regime is extremely restrictive: in reality, large-scale atmospheric and oceanic flows contain a number of mutually interacting dynamical regimes, even within the context of slow dynamics. An atmospheric example is the development of a semi-geostrophic front within a quasi-geostrophic baroclinic disturbance. An oceanic example is the development of quasi-geostrophic eddies within a planetary geostrophic gyre circulation. Yet nobody has been able to derive a balanced model capable of describing multiple dynamical regimes within the framework of a formal asymptotic theory.

An alternative approach to obtaining balanced models is to impose a set of dynamical constraints a priori, based on observed characteristics of the flow. (The distinction between this approach and the asymptotic one is not, perhaps, as great as it might seem: any choice of small parameters, including the assumption of slow dynamics, is similarly based on observed characteristics of the flow.) Probably the best-known example of such a model is the three-dimensional $f$-plane semi-geostrophic (SG) model of Hoskins (1975), which has been widely used in meteorology. A particularly attractive feature of the SG model is that for small Rossby and Froude numbers (and weak topography) it reduces to ( $f$-plane) QG dynamics, while for anisotropic flows it reduces to two-dimensional SG dynamics; thus it can describe the formation of fronts within developing QG baroclinic systems, provided the fronts are not strongly curved. The SG model also describes the dynamics of low-frequency coastally trapped waves (Allen, Barth \& Newberger 1990a; Kushner, McIntyre \& Shepherd 1997), which obey anisotropic scaling and are thus semi-geostrophic provided the coast is not strongly curved (see also 2 ).

In deriving reduced models, it is arguable (Lorenz 1960; Sadourny 1975; Salmon 1983) that one should preserve the fundamental symmetry-based conservation laws of the underlying primitive equations. Such conservation laws underpin much of our theoretical understanding of dynamical models, and provide a unifying link between different models. This is particularly the case for the considerable body of theory concerning disturbances to prescribed basic states, including available potential energy, wave-activity invariants, and stability theorems (e.g. Holm et al. 1985; McIntyre \& Shepherd 1987; Shepherd 1990, 1993). In the case of stability theorems, for example, it turns out that virtually all of the classical inviscid linear stability theorems (e.g. static stability, symmetric stability, Rayleigh's inflection-point and centrifugal-stability theorems, and the Fjørtoft-Pedlosky and Charney-Stern theorems) may be understood in this broader context, and may furthermore be extended to finite amplitude (see e.g. Shepherd 1994). One can argue that leading-order asymptotic approximations 
will inevitably preserve symmetry-based conservation laws; this argument has been formalized by Bokhove (1996, Chapter 4). For constrained models no such property is guaranteed, unless the constraints are imposed within the context of a Hamiltonian description of the dynamics (Salmon 1983). Of course, Hamiltonian structure in itself is no guarantee of a model's accuracy.

Salmon (1985) showed how the $f$-plane SG model could be derived within a Hamiltonian framework, and could be generalized to include variable Coriolis parameter (see also Shutts 1989; Magnusdottir \& Schubert 1990, 1991). By exploiting the relevant conservation laws, Kushner \& Shepherd $(1995 a, b)$ were able to extend much of the disturbance theory that exists for QG dynamics to SG dynamics. (They considered the $f$-plane SG model as well as the $\beta$-plane compressible SG model of Magnusdottir \& Schubert 1990.) One of the novel features of the SG theory is the appearance of lateral boundary contributions to the wave-activity invariants, which affect the stability theorems. These lateral boundary contributions are associated with the presence of coastally trapped waves, which are filtered in the QG model. Kushner et al. (1997) have shown that the impact of the boundary terms on the stability theorems reflects a genuine physical instability mechanism associated with these coastal waves.

The work of Kushner \& Shepherd $(1995 a, b)$ appears to be the first to deal with lateral boundary contributions to wave-activity invariants in a balanced model, and with their effect on stability theorems. However, the SG model has a number of complicating features and the analysis of Kushner \& Shepherd is mathematically quite tortuous. In particular, the SG model is most naturally formulated in a transformed coordinate system, but this transformation produces moving boundaries. In addition, the SG invertibility relation between geostrophic velocity and potential vorticity is extremely complicated. As a consequence, Kushner \& Shepherd had only limited success in extending their small-amplitude results to finite amplitude. It was not clear whether this difficulty was merely technical, or whether it reflected something deeper. Moreover, the way in which lateral boundary contributions arise within the SG wave-activity invariants remains somewhat mysterious (see Kushner \& Shepherd $1995 b, \S 6)$.

It is therefore of interest to examine the nature of lateral boundary contributions to wave-activity invariants within the context of a constrained balanced model that has simpler mathematical properties than the SG model yet contains the essential physics. Salmon's (1983) model of nearly geostrophic dynamics is a natural choice for such a study. Like the SG model, it describes multiple dynamical regimes: quasi-geostrophic dynamics, planetary geostrophic dynamics, anisotropic semi-geostrophic dynamics, and low-frequency coastally trapped waves. Since it is derived within a Hamiltonian framework, it automatically possesses appropriate symmetry-based conservation laws. The model may be formulated for stratified flow, but to simplify the analysis (in order to focus on the physics) we follow Salmon (1983) and restrict attention to the shallow-water context.

It has recently become evident that Salmon's (1983) model is an important prototype for constrained Hamiltonian balanced models. The model is derived by constraining the velocity to the mass field within the framework of Hamilton's principle. As a consequence, the advecting velocity differs from the (constraint) velocity that appears in the conservation laws. (This is a property of many reduced systems, including the hydrostatic equations.) In Salmon's (1983) model the constraint velocity is taken to be the geostrophic velocity, but it is clear from Salmon's construction that other (potentially more accurate) choices are also possible. Both Allen \& Holm (1996) and 
McIntyre \& Roulstone (1997) have recently developed this idea and proposed general theories for deriving constrained Hamiltonian balanced models that explicitly rely on just such a velocity 'split'. Salmon's (1983) model - referred to in those studies as 'HP dynamics' and ' $\mathrm{L}_{1}$ dynamics', respectively - turns out to occupy a central role in both theories.

In this paper, we derive wave-activity invariants for Salmon's (1983) model and use those invariants to obtain nonlinear stability theorems. The model is described in $\$ 2$, including a clarification of the lateral boundary conditions. The analysis then follows, first for general steady basic flows ( $\$ 3)$, and then for steady basic flows having zonal or rotational symmetry $(\S 4)$. It is shown that Salmon's model possesses an analogue of Ripa's (1983) small-amplitude stability theorem for shallow-water flow, as well as finite-amplitude analogues of Kushner \& Shepherd's $(1995 a, b)$ SG stability theorems. In the process, the way in which the geostrophic constraint produces boundary contributions to wave-activity invariants is clarified. The implications for more general constrained Hamiltonian balanced models are discussed in $§ 5$.

\section{Governing equations and boundary conditions}

For the purpose of this paper, the 'primitive' equations are taken to be the rotating shallow-water equations; this system arguably represents the simplest model in geophysical fluid dynamics that contains the essential physics of nonlinear vortical motion coupled to fast (in this case inertia-gravity) waves. The governing equations are given by (e.g. Pedlosky 1987)

$$
\begin{gathered}
\frac{\partial \boldsymbol{u}}{\partial t}+q h \hat{\boldsymbol{z}} \times \boldsymbol{u}=-\nabla\left(g \eta+\frac{1}{2}|\boldsymbol{u}|^{2}\right), \\
\frac{\partial h}{\partial t}+\nabla \cdot(h \boldsymbol{u})=0,
\end{gathered}
$$

where $\boldsymbol{u}(x, y, t)$ is the (horizontal) velocity, $\eta(x, y, t)$ is the free-surface height, $h(x, y, t)=$ $\eta-h_{B}$ is the fluid depth, $h_{B}(x, y)$ is the bottom topography, $q \equiv[f+\hat{z} \cdot(\nabla \times \boldsymbol{u})] / h$ is the potential vorticity, $g$ is the (constant) gravitational acceleration, $f(y)$ is the Coriolis parameter, $\hat{z}$ is the unit vertical vector, and $\nabla \equiv(\partial / \partial x, \partial / \partial y) \equiv\left(\partial_{x}, \partial_{y}\right)$, where $x$ and $y$ are respectively eastward (zonal) and northward (meridional) coordinates. Various domain geometries $D$ may be considered: closed, open, or periodic in one or two directions. In the case of an open (unbounded) domain, appropriate decay conditions are assumed to apply at infinity. In the case of a periodic domain, all integral quantities are averaged in the periodic coordinate. At any rigid boundary, the boundary condition is simply that of no normal flow:

$$
\boldsymbol{u} \cdot \hat{\boldsymbol{n}}=0 \quad \text { on } \partial D \quad(\hat{\boldsymbol{n}}=\text { outward normal to boundary } \partial D) .
$$

This system conserves an energy

$$
\mathscr{H}=\int \frac{1}{2}\left(h|\boldsymbol{u}|^{2}+g \eta^{2}\right) \mathrm{d} \boldsymbol{x},
$$

as well as a family of Casimirs

$$
\mathscr{C}=\int h C(q) \mathrm{d} \boldsymbol{x},
$$

where $C(\cdot)$ is an arbitrary function. The Casimir invariants are a consequence of the material conservation of potential vorticity, $q_{t}+\boldsymbol{u} \cdot \nabla q=0$, which follows from (2.1); 
the total mass invariant $\int h \mathrm{~d} \boldsymbol{x}$ corresponds to $C \equiv 1$. When the domain (boundaries and bottom topography) is zonally symmetric, then the system conserves a zonal absolute momentum

$$
\mathscr{M}=\int h(u-F(y)) \mathrm{d} \boldsymbol{x},
$$

where $\boldsymbol{u} \equiv(u, v)$ and $F(y) \equiv \int f(y) \mathrm{d} y$; the constant of integration for $F$ is irrelevant in (2.5), because of conservation of total mass. When the domain is rotationally symmetric (this requires $f(y)=$ const) then the system conserves an absolute angular momentum

$$
\mathscr{J}=\int h\left(w+\frac{1}{2} f r^{2}\right) \mathrm{d} \boldsymbol{x},
$$

where $w \equiv \hat{z} \cdot(\boldsymbol{x} \times \boldsymbol{u})$ and $r^{2}=x^{2}+y^{2}$. Finally, using (2.1a) together with (2.2) one can derive conservation of circulation on each connected (either closed or periodic) piece $\partial D_{i}$ of the boundary:

$$
\frac{\mathrm{d} \Gamma_{i}}{\mathrm{~d} t} \equiv \frac{\mathrm{d}}{\mathrm{d} t} \oint_{\partial D_{i}} \boldsymbol{u} \cdot \mathrm{d} \ell=0
$$

where $\mathrm{d} \ell$ is tangent to the boundary, oriented so that $\hat{\boldsymbol{n}}$ is on the right.

Salmon's (1983) model retains all the above conservation laws, with the full velocity $\boldsymbol{u}$ replaced in all expressions (including that for $q$ ) by the geostrophic velocity

$$
\boldsymbol{u}_{G} \equiv \frac{g}{f} \hat{z} \times \nabla \eta=\frac{g}{f} \hat{z} \times \nabla\left(h+h_{B}\right) .
$$

These conservation laws are enforced by setting $\boldsymbol{u}=\boldsymbol{u}_{G}$ within the context of Hamilton's principle. (Salmon 1983 only explicitly considered $\mathscr{H}$ and $\mathscr{C}$, but conservation of $\mathscr{M}, \mathscr{J}$, and $\Gamma_{i}$ also follows.) Note that in order for Salmon's model to be physically meaningful, we must have $f \neq 0$ everywhere in the domain. The evolution equations that result from this procedure $\dagger$ are the full mass equation (2.1b), but with $(2.1 a)$ replaced by

$$
\frac{\partial \boldsymbol{u}_{G}}{\partial t}+q h \hat{\boldsymbol{z}} \times \boldsymbol{u}=-\nabla\left[g \eta+\frac{1}{2}\left|\boldsymbol{u}_{G}\right|^{2}+\hat{z} \cdot \nabla \times\left(\frac{g h \boldsymbol{u}_{A G}}{f}\right)\right],
$$

where $\boldsymbol{u}_{A G} \equiv \boldsymbol{u}-\boldsymbol{u}_{G}$ is the ageostrophic velocity, and now $q \equiv\left[f+\hat{\boldsymbol{z}} \cdot\left(\nabla \times \boldsymbol{u}_{G}\right)\right] / h$. It should be noted that the two scalar equations represented by (2.9) are not independent prognostic (evolution) equations; because $\boldsymbol{u}_{G}$ is determined by $h$, only one of the three equations $(2.1 b)$ and (2.9) can be used as a prognostic equation, with the other two providing the constraints required to determine $\boldsymbol{u}_{A G}$ (see Appendix A). Thus Salmon's model is a balanced model, of first order in time; this reflects the fact that the inertia-gravity wave solutions of shallow-water dynamics have been eliminated.

In order to provide a unique determination of $\boldsymbol{u}_{A G}$ in the presence of rigid boundaries, another boundary condition on the velocity is required in addition to (2.2) (see Appendix A). Allen et al. (1990a) propose

$$
\boldsymbol{u}_{A G} \times \hat{\boldsymbol{n}}=0 \quad \text { on } \partial D \quad(\hat{\boldsymbol{n}}=\text { outward normal to boundary } \partial D),
$$

which is sufficient (together with periodicity or decay conditions as the case may be) to enforce conservation of (the geostrophic version of) $\mathscr{H}$. The condition (2.10) may be deduced directly from Hamilton's principle (R. Salmon, unpublished notes; Allen \& Holm 1996), and not surprisingly, therefore, it is also sufficient to enforce 
conservation of $\mathscr{M}, \mathscr{J}$, and $\Gamma_{i}$. ( $\mathscr{C}$ is automatically conserved by virtue of (2.2).) However, it is important to determine the conditions under which (2.10) is accurate. The component of the primitive momentum equation (2.1a) normal to the boundary can be written

$$
-V \frac{\mathrm{D} \chi}{\mathrm{D} t}+f V=g \hat{\boldsymbol{n}} \cdot \nabla \eta,
$$

where $V$ is the tangential velocity, $\chi$ is the angle the boundary (and hence the flow) makes with a fixed direction, and $\mathrm{D} / \mathrm{D} t$ is the material derivative. For straight boundaries, $\mathrm{D} \chi / \mathrm{D} t=0$ and the tangential flow is therefore strictly geostrophic, making (2.10) exact. For curved boundaries, $\mathrm{D} \chi / \mathrm{D} t=-V / R$ where $R$ is the local radius of curvature of the boundary. Thus (2.10) provides a good approximation to (2.11) whenever $|V| / f R \ll 1$. This weak-curvature condition is precisely that under which the SG approximation is valid (Hoskins 1975).

Note that $\boldsymbol{u}_{G}$ is not required to be tangent to rigid boundaries; only $\boldsymbol{u}$ is. This corresponds to the situation with SG dynamics (Kushner \& Shepherd 1995a), and is an important distinguishing feature with respect to QG dynamics. In particular, this feature allows the representation of coastal Kelvin waves, which are filtered in QG dynamics. Since coastal Kelvin waves are semi-geostrophic in the literal sense of being geostrophically balanced in one direction, it is appropriate that they should be represented in these models.

\section{Steady basic flows without presumed spatial symmetry}

For Hamiltonian systems, it is well known that steady states are constrained extremals of the energy, the constraint being imposed through a suitable Casimir. Thus one may generally construct exact finite-amplitude invariants that are quadratic to leading order in the disturbance to a given steady state. Following McIntyre \& Shepherd (1987), we refer to disturbance invariants having this general property as wave-activity invariants; in the special case considered in this section, the wave-activity invariant is known variously as the free energy, pseudoenergy, or energy-Casimir invariant. See Holm et al. (1985) or Shepherd (1990) for further background.

The application of this method to Salmon's model turns out to be straightforward, so technical details are kept to a minimum. We consider disturbances $\left(\boldsymbol{u}_{G}^{\prime}, \boldsymbol{u}_{A G}^{\prime}, h^{\prime}\right)$ to a steady basic flow $\left(\boldsymbol{U}_{G}, \boldsymbol{U}_{A G}, H\right)$; hence $h=H+h^{\prime}$, etc. The basic flow is presumed to be a steady solution of Salmon's model. Thus $(2.1 b)$ implies $\nabla \cdot H \boldsymbol{U}=0$, and one may introduce a mass stream function $\Psi$ defined by $H \boldsymbol{U}=\hat{z} \times \nabla \Psi$. Then (2.9) implies

$$
Q \nabla \Psi=\nabla B,
$$

where $B$ and $Q$ denote the generalized Bernoulli function and potential vorticity evaluated at the steady basic flow, namely

$$
\begin{gathered}
B \equiv g N+\frac{1}{2}\left|\boldsymbol{U}_{G}\right|^{2}+\hat{z} \cdot \nabla \times\left(\frac{g H \boldsymbol{U}_{A G}}{f}\right), \\
Q \equiv \frac{f+\hat{z} \cdot\left(\nabla \times \boldsymbol{U}_{G}\right)}{H},
\end{gathered}
$$

with $N \equiv H+h_{B}$.

In the usual way, the energy, Casimir, and circulation invariants of Salmon's model 
may be combined to construct the exact invariant

$$
\mathscr{A}[H ; h] \equiv\left(\mathscr{H}+\mathscr{C}+\sum_{i} \lambda_{i} \Gamma_{i}\right)[h]-\left(\mathscr{H}+\mathscr{C}+\sum_{i} \lambda_{i} \Gamma_{i}\right)[H] .
$$

In (3.3), the sum over $i$ runs over all connected pieces $\partial D_{i}$ of the boundary, and $\lambda_{i}$ are free parameters; all conserved quantities are functionals of the height field alone, in the light of (2.8) and (A 1)-(A 2), so we may write $\mathscr{H}[h]$, etc. The form of the Casimir and the parameters $\lambda_{i}$ are determined by the choice of the basic flow through the extremal condition $\delta \mathscr{A}=0$. This may be seen (see Appendix B) to lead to

$$
C(\xi)=\xi\left(\int_{0}^{\xi} \frac{K(\gamma)}{\gamma^{2}} \mathrm{~d} \gamma+c\right) \quad(c \text { is an arbitrary constant })
$$

and

$$
\lambda_{i}=-\left.C^{\prime}(Q)\right|_{\partial D_{i}}
$$

where the function $K(\cdot)$ is defined by

$$
B(x, y)=K(Q(x, y)) \quad \forall(x, y) .
$$

Since both $B$ and $Q$ are constant along lines of constant $\Psi$ (this follows from (3.1) and the fact that $\boldsymbol{U} \cdot \nabla Q=0$ ), the function $K(\cdot)$ is well defined by (3.6) provided $\nabla Q \neq 0$ almost everywhere. (The case where $Q(x, y)$ is piecewise constant would require a different approach.) In general, the function $K(\cdot)$ so defined will be multivalued, but our pseudoenergy-based stability theorems will apply only to single-valued $K(Q)$ so this issue will not concern us. The condition (3.5) can likewise be satisfied, because each connected piece of the boundary must be a line of constant $\Psi$ and thus a line of constant $Q$.

There is one non-trivial technical detail that should be mentioned at this point, because it has implications for more general constrained Hamiltonian balanced models. Steady flows in Salmon's model must generally have $\boldsymbol{U}_{A G} \neq 0$, unless the flow is straight. (To see this, suppose a steady flow was strictly geostrophic. Then (2.9) would reduce to $(2.1 a)$ with $\boldsymbol{U}$ replaced by $\boldsymbol{U}_{G}$. But then (2.11) would apply at all points in the domain, with $V$ equal to the geostrophic velocity and $\hat{\boldsymbol{n}}$ normal to the flow. This would imply $\mathrm{D} \chi / \mathrm{D} t=0$, namely straight flow.) But then $\delta C$ involves $\boldsymbol{U}_{A G}$, while $\delta\left(\mathscr{H}+\sum_{i} \lambda_{i} \Gamma_{i}\right)$ does not, and it is not obvious that the extremal condition will be satisfied. This issue can be expected to be generic for velocity-split models (see §5). However, if one computes $\delta \mathscr{A}$ with (3.4) and (3.5), noting from (3.6) that

$$
C^{\prime}(Q)=\frac{C+B}{Q} \quad \text { and } \quad \nabla C^{\prime}(Q)=\frac{\nabla B}{Q},
$$

then after using (3.1) and noting the cancellation of the terms involving $N$ and $\boldsymbol{U}_{G}$ one is left with

$$
\begin{aligned}
\delta \mathscr{A} & =\int\left\{-B_{A G} \delta h-\hat{z} \cdot\left(\nabla \Psi_{A G} \times \delta \boldsymbol{u}_{G}\right)\right\} \mathrm{d} \boldsymbol{x} \\
& =\int\left\{-B_{A G}+\nabla \cdot\left(\frac{g}{f} \nabla \Psi_{A G}\right)\right\} \delta h \mathrm{~d} \boldsymbol{x}-\sum_{i} \oint_{\partial D_{i}} \frac{g}{f} \nabla \Psi_{A G} \cdot \hat{\boldsymbol{n}} \delta h \mathrm{~d} s,
\end{aligned}
$$

where $B_{A G}$ is the difference between $B$ and the usual Bernoulli function evaluated for the geostrophic flow (i.e. the first two terms of (3.2a)), and $\Psi_{A G}$ is defined by $H U_{A G}=\hat{z} \times \nabla \Psi_{A G}$. In order for the interior terms in (3.8b) to vanish for arbitrary $\delta h$ 
we must have $B_{A G}=-\nabla \cdot\left((g / f) \hat{z} \times H U_{A G}\right)$, which is precisely the extra (ageostrophic) term in $(3.2 a)$. As for the boundary term in $(3.8 b)$, it vanishes for arbitrary $\delta h$ by (2.10). Thus the ageostrophic contribution to the generalized Bernoulli function (3.2a) and the boundary condition (2.10) are precisely what is required for steady flows to be constrained extremals of $\mathscr{H}$ in Salmon's model. Of course, this is no accident; it is a consequence of the Hamiltonian structure of the model.

With these choices of $C(\cdot)$ and $\lambda_{i}$, the finite-amplitude pseudoenergy (3.3) can be written in the form

$$
\mathscr{A}=\int\left\{\frac{1}{2}\left(H+h^{\prime}\right)\left|\boldsymbol{u}_{G}^{\prime}\right|^{2}+h^{\prime} \boldsymbol{U}_{G} \cdot \boldsymbol{u}_{G}^{\prime}+\frac{1}{2} g\left(h^{\prime}\right)^{2}+\left(H+h^{\prime}\right) \int_{0}^{q^{\prime}}\left[C^{\prime}(Q+\xi)-C^{\prime}(Q)\right] \mathrm{d} \xi\right\} \mathrm{d} \boldsymbol{x},
$$

with the disturbance potential vorticity given by

$$
q^{\prime} \equiv q-Q=\frac{\hat{z} \cdot\left(\nabla \times \boldsymbol{u}_{G}^{\prime}\right)-Q h^{\prime}}{H+h^{\prime}} .
$$

Left in this form, $\mathscr{A}$ is identical to the pseudoenergy for the shallow-water equations (Shepherd 1992), with $\boldsymbol{U}$ and $\boldsymbol{u}^{\prime}$ replaced by $\boldsymbol{U}_{G}$ and $\boldsymbol{u}_{G}^{\prime}$. Following Ripa (1983), (3.9) may be re-written as

$$
\begin{aligned}
\mathscr{A}=\int\left\{\frac{1}{2\left(H+h^{\prime}\right)} \mid\left(H+h^{\prime}\right) \boldsymbol{u}_{G}^{\prime}\right. & +\left.\boldsymbol{U}_{G} h^{\prime}\right|^{2}+\frac{1}{2}\left(g-\frac{\left|\boldsymbol{U}_{G}\right|^{2}}{H+h^{\prime}}\right)\left(h^{\prime}\right)^{2} \\
& \left.+\left(H+h^{\prime}\right) \int_{0}^{q^{\prime}}\left[C^{\prime}(Q+\xi)-C^{\prime}(Q)\right] \mathrm{d} \xi\right\} \mathrm{d} \boldsymbol{x} .
\end{aligned}
$$

The small-amplitude (quadratic) approximation to (3.11) is seen to be positive definite for arbitrary disturbances whenever

$$
\left|\boldsymbol{U}_{G}\right|^{2}<g H
$$

and

$$
C^{\prime \prime}(Q)=\frac{\nabla B}{Q \nabla Q}=\frac{\nabla \Psi}{\nabla Q}>0,
$$

which is the analogue of Ripa's (1983) shallow-water stability theorem for Salmon's model. Condition (3.12b) corresponds to that of Arnol'd's (1966) first stability theorem, and is a sufficient condition for stability in the QG model. Condition (3.12a) corresponds to a 'subsonic' condition (Ripa 1983), in the sense that the (geostrophic) basic flow speed must be everywhere less than the minimum gravity-wave phase speed. The physical interpretation of Ripa's theorem in the shallow-water case is that $(3.12 b)$ ensures stability of the balanced dynamics, while $(3.12 a)$ ensures that the gravity waves cannot interact with the balanced dynamics; Ford (1993, Chapter 3) has shown for a particular parallel jet flow that when the latter condition is violated, a coupled vortical/gravity-wave instability arises. (See also Nore \& Shepherd 1997, §7.) Although Salmon's model eliminates the inertia-gravity waves, it retains the coastal Kelvin waves which also have phase speeds greater than or equal to $(g H)^{1 / 2}$ (Allen et al. 1990a).

There are two important limitations of Ripa's (1983) theorem. The first is that it does not generalize to continuously stratified flow (Ripa 1991). The second is that it does not extend to finite amplitude, since from (3.11) the subsonic condition would then have to hold with respect to the actual fluid depth $H+h^{\prime}$, and there is nothing in the dynamics to prevent $H+h^{\prime}$ from becoming arbitrarily small (Shepherd 1992). 
A notable feature of (3.9) is that it does not contain any boundary terms. However, we have not exploited the fact that $\boldsymbol{u}_{G}^{\prime}$ and $h^{\prime}$ are related by $f \boldsymbol{u}_{G}^{\prime}=g \hat{z} \times \nabla h^{\prime}$. Applying this constraint, (3.9) becomes

$$
\begin{aligned}
\mathscr{A}= & \int\left\{\frac{g^{2}}{2 f^{2}}\left(H+h^{\prime}\right)\left|\nabla h^{\prime}\right|^{2}+\frac{1}{2}\left[g-\nabla \cdot\left(\frac{g^{2}}{f^{2}} \nabla N\right)\right]\left(h^{\prime}\right)^{2}\right. \\
& \left.+\left(H+h^{\prime}\right) \int_{0}^{q^{\prime}}\left[C^{\prime}(Q+\xi)-C^{\prime}(Q)\right] \mathrm{d} \xi\right\} \mathrm{d} \boldsymbol{x}+\sum_{i} \oint_{\partial D_{i}} \frac{g^{2}}{2 f^{2}} \nabla N \cdot \hat{\boldsymbol{n}}\left(h^{\prime}\right)^{2} \mathrm{~d} s .
\end{aligned}
$$

We thus see that applying the geostrophic constraint produces a boundary contribution to the pseudoenergy. Such a boundary contribution is also found in SG dynamics (Kushner \& Shepherd 1995b), but not in QG dynamics (McIntyre \& Shepherd 1987).

In contrast to (3.11), the form (3.13) of the pseudoenergy may be used to derive a nonlinear stability theorem. In particular, $\mathscr{A}$ is seen to be positive definite and convex whenever there exist constants $c_{1}, c_{2}, k_{1}, k_{2}, b_{1}^{(i)}$ and $b_{2}^{(i)}$ such that

$$
\begin{gathered}
0<c_{1} \leqslant C^{\prime \prime}(Q)=\frac{\nabla \Psi}{\nabla Q} \leqslant c_{2}<\infty, \\
0<k_{1} \leqslant f-\zeta_{G}-\frac{U_{G}}{f} \frac{\mathrm{d} f}{\mathrm{~d} y} \leqslant k_{2}<\infty, \\
0<b_{1}^{(i)} \leqslant \frac{g}{f} \nabla N \cdot \hat{\boldsymbol{n}} \leqslant b_{2}^{(i)}<\infty \quad \text { on } \partial D_{i},
\end{gathered}
$$

where $\zeta_{G} \equiv \hat{z} \cdot\left(\nabla \times \boldsymbol{U}_{G}\right)$ and $U_{G} \equiv-(g / f) \mathrm{d} N / \mathrm{d} y$. Defining new disturbance variables according to

$$
\tilde{q} \equiv\left(H+h^{\prime}\right)^{1 / 2} q^{\prime}, \quad \boldsymbol{m} \equiv\left(H+h^{\prime}\right)^{1 / 2} \nabla h^{\prime}
$$

(this transformation is always smooth since $h=H+h^{\prime}>0$ ), and introducing the disturbance norm $\dagger$

$$
\left\|h^{\prime}\right\|^{2} \equiv \int \frac{1}{2}\left\{\frac{g^{2}}{f^{2}}|\boldsymbol{m}|^{2}+\frac{g}{f} k_{1}\left(h^{\prime}\right)^{2}+c_{1} \tilde{q}^{2}\right\} \mathrm{d} \boldsymbol{x}+\sum_{i} \oint_{\partial D_{i}} \frac{g b_{1}^{(i)}}{2 f}\left(h^{\prime}\right)^{2} \mathrm{~d} s
$$

(note that $f \neq 0$ by hypothesis), it follows that

$$
\left\|h^{\prime}(t)\right\|^{2} \leqslant \mathscr{A}(t)=\mathscr{A}(0) \leqslant \max \left\{\frac{c_{2}}{c_{1}}, \frac{k_{2}}{k_{1}}, \frac{b_{2}^{(i)}}{b_{1}^{(i)}}\right\}\left\|h^{\prime}(0)\right\|^{2} .
$$

The inequalities (3.17) establish nonlinear Liapunov stability of any steady basic flow satisfying (3.14).

We now consider the interpretation of the stability conditions (3.14). Condition (3.14a), like (3.12b), corresponds to that of Arnol'd's (1966) first stability theorem. Condition (3.14b), which ensures positivity of the factor multiplying $\left(h^{\prime}\right)^{2}$ in $(3.13)$, is assured of being true if the basic flow has small Rossby number. Finally, condition (3.14c) requires that the basic flow be cyclonic on lateral boundaries. This condition is the same as that found for the SG model (Kushner \& Shepherd 1995b). As discussed by Kushner \& Shepherd, the combination of (3.14a) and (3.14c) can be understood in terms of the possible interactions between interior disturbances and coastal Kelvin waves. Kushner et al. (1997) present an example (in the context of

$\dagger$ This is not a norm in $h^{\prime}$, but rather in the composite variables $\left(\boldsymbol{m}, h^{\prime}, \tilde{q}\right)$; nevertheless we write $\left\|h^{\prime}\right\|$ since (3.16) is a functional of $h^{\prime}$. 
the stratified SG model) where violation of condition (3.14c) leads to a phase-locked instability between counterpropagating coastal Kelvin waves on opposite walls of a channel.

Thus we see that Salmon's model possesses two distinct kinds of stability theorems. The first, corresponding to conditions (3.12), is analogous to Ripa's shallow-water theorem, and does not extend to finite amplitude. The second, corresponding to conditions (3.14), is analogous to the SG theorem of Kushner \& Shepherd (1995b), and does extend to finite amplitude. In both cases, the stability conditions consist of Arnol'd's condition plus an additional condition that prevents phase-locked coupling between coastal Kelvin waves and the rest of the dynamics (either the interior dynamics, or other coastal Kelvin waves if there is more than one lateral boundary). The subsonic condition (3.12a) is sufficient for this, but is too crude: it does not take account of the fact that the only gravity waves in Salmon's model are boundarytrapped (so the subsonic condition should only be required at the boundaries), or the fact that these waves are unidirectional. These two facts are reflected in the cyclonic flow condition (3.14c), which ensures that the basic flow at the boundaries acts to speed up rather than slow down the coastal Kelvin waves. However this condition is also too crude, insofar as anticyclonic flow at the boundaries should not be destabilizing so long as it is subsonic.

That the stability criteria of the two theorems are indeed complementary is illustrated by the following example. Consider a periodic zonal channel $-Y \leqslant y \leqslant Y$ on an $f$-plane, with the basic flow

$$
U_{G}(y)=u_{0} \sinh \lambda y, \quad H(y)=H_{0}-\gamma \cosh \lambda y-\frac{f u_{0}}{g \lambda} \cosh \lambda y .
$$

First consider the case $u_{0}>0$ and $\gamma=0$, corresponding to anticyclonic flow over a flat bottom. Then condition $(3.14 c)$ does not apply. But it is easy to see that for sufficiently large $H_{0}$ (other parameters being fixed), this basic flow can satisfy conditions $(3.12 a, b)$. Now consider the case $u_{0}<0$ and $\gamma=f\left|u_{0}\right| / g \lambda$, corresponding to cyclonic flow along a valley. Then $H(y)=H_{0}$, and conditions (3.14a) and (3.14c) hold automatically. Condition (3.14b) will hold if

$$
\left|u_{0}\right| \lambda \cosh \lambda y<f
$$

for all $y$, while condition $(3.12 a)$ will fail if

$$
u_{0}^{2} \sinh ^{2} \lambda y>g H_{0}
$$

for some $y$. It is enough for the inequalities (3.19) and (3.20) to be satisfied at the channel wall $y=Y$, which can be made to happen (for appropriate choices of $u_{0}$ and $Y)$ provided $\lambda<f /\left(g H_{0}\right)^{1 / 2}$. This latter condition corresponds to the lengthscale of the basic flow being greater than the Rossby deformation radius, which is precisely what is required in order to have the Froude number greater than unity, namely (3.20), while keeping the Rossby number less than unity, namely (3.19).

We have therefore shown that there exist basic flows satisfying (3.12) but not (3.14), as well as basic flows satisfying (3.14) but not (3.12); the two stability theorems have overlapping but distinct criteria. However, the theorem corresponding to (3.14) has the advantage of being fully nonlinear. 


\section{Steady basic flows with spatial symmetry}

\subsection{Zonally symmetric basic flows}

We first consider the case where the basic flow (and domain geometry) is zonally symmetric, i.e. $\partial / \partial x=0$ for all basic-flow fields and $\partial h_{B} / \partial x=0$. In this case, the basic flow must be zonal and geostrophic. (The latter follows from (A 2) and (2.10).) We can now include the momentum invariant of Salmon's model together with the energy, Casimir and circulation invariants to produce the wave-activity invariant

$$
\mathscr{A}[H ; h] \equiv\left(\mathscr{H}+\alpha \mathscr{M}+\mathscr{C}+\sum_{i} \lambda_{i} \Gamma_{i}\right)[h]-\left(\mathscr{H}+\alpha \mathscr{M}+\mathscr{C}+\sum_{i} \lambda_{i} \Gamma_{i}\right)[H],
$$

where $\alpha$ is a free parameter. The extremal condition $\delta \mathscr{A}=0$ may be seen (see Appendix B) to be satisfied by the choice

$$
C(\xi)=\xi\left(\int_{0}^{\xi} \frac{[K+\alpha G](\gamma)}{\gamma^{2}} \mathrm{~d} \gamma+c\right) \quad(c \text { is an arbitrary constant })
$$

as well as (3.5), where the (possibly multivalued) function $G(\cdot)$ is defined by $G(Q(y))=$ $U_{G}(y)-F(y)$ for all $y$. At the basic state, we then have

$$
C^{\prime}(Q)=\frac{C+B+\alpha\left(U_{G}-F\right)}{Q} \quad \text { and } \quad \frac{\mathrm{d}}{\mathrm{d} y} C^{\prime}(Q)=\frac{B_{y}}{Q}-\alpha H,
$$

and (4.1) takes the form

$\mathscr{A}=\int\left\{\frac{1}{2}\left(H+h^{\prime}\right)\left|\boldsymbol{u}_{G}^{\prime}\right|^{2}+h^{\prime}\left(U_{G}+\alpha\right) u_{G}^{\prime}+\frac{1}{2} g\left(h^{\prime}\right)^{2}+\left(H+h^{\prime}\right) \int_{0}^{q^{\prime}}\left[C^{\prime}(Q+\xi)-C^{\prime}(Q)\right] \mathrm{d} \xi\right\} \mathrm{d} \boldsymbol{x}$.

As with (3.9), we may obtain an analogue of Ripa's (1983) small-amplitude stability theorem from (4.4): the basic flow is stable if a value of $\alpha$ can be found for which

$$
\left(U_{G}+\alpha\right)^{2}<g H
$$

and

$$
C^{\prime \prime}(Q)=-\frac{U_{G}+\alpha}{Q_{y}}>0 .
$$

These conditions correspond to $(3.12 a, b)$ applied to a geostrophic zonal basic flow, allowing for an arbitrary Galilean boost to $U_{G}$.

The form (4.4) of the wave-activity invariant involves no boundary contributions. However, boundary contributions are produced upon using the geostrophic constraint, which yields

$$
\begin{aligned}
\mathscr{A}= & \int\left\{\frac{g^{2}}{2 f^{2}}\left(H+h^{\prime}\right)\left|\nabla h^{\prime}\right|^{2}+\frac{1}{2}\left[g-\frac{\mathrm{d}}{\mathrm{d} y}\left(\frac{g^{2}}{f^{2}} \frac{\mathrm{d} N}{\mathrm{~d} y}\right)-\alpha \frac{g}{f^{2}} \frac{\mathrm{d} f}{\mathrm{~d} y}\right]\left(h^{\prime}\right)^{2}\right. \\
& \left.+\left(H+h^{\prime}\right) \int_{0}^{q^{\prime}}\left[C^{\prime}(Q+\xi)-C^{\prime}(Q)\right] \mathrm{d} \xi\right\} \mathrm{d} \boldsymbol{x}-\sum_{i} \oint_{\partial D_{i}} \frac{g}{2 f}\left(U_{G}+\alpha\right) \hat{\boldsymbol{n}} \cdot \hat{\boldsymbol{y}}\left(h^{\prime}\right)^{2} \mathrm{~d} s .
\end{aligned}
$$

Evidently $\mathscr{A}$ is positive definite and convex whenever there exists a value of $\alpha$ and 
constants $c_{1}, c_{2}, k_{1}, k_{2}, b_{1}^{(i)}$ and $b_{2}^{(i)}$ such that

$$
\begin{gathered}
0<c_{1} \leqslant C^{\prime \prime}(Q)=-\frac{H\left(U_{G}+\alpha\right)}{Q_{y}} \leqslant c_{2}<\infty, \\
0<k_{1} \leqslant f-\zeta_{G}-\frac{\left(U_{G}+\alpha\right)}{f} \frac{\mathrm{d} f}{\mathrm{~d} y} \leqslant k_{2}<\infty, \\
0<b_{1}^{(i)} \leqslant-\left(U_{G}+\alpha\right) \hat{\boldsymbol{n}} \cdot \hat{\boldsymbol{y}} \leqslant b_{2}^{(i)}<\infty \quad \text { on } \partial D_{i} .
\end{gathered}
$$

These three conditions correspond to the pseudoenergy-based stability conditions (3.14) applied to a geostrophic zonal basic flow, allowing for an arbitrary Galilean boost to $U_{G}$. Defining a disturbance norm by (3.16), the inequalities (3.17) again follow. This establishes nonlinear stability of any steady zonally symmetric geostrophic basic flow satisfying (4.7) for some $\alpha$.

It is interesting to consider what happens when the energy is not used in the wave-activity construction (4.1), in which case $\mathscr{A}$ is just the pseudomomentum. The results may be formally extracted from (4.2)-(4.4) by taking the limit $\alpha \rightarrow \pm \infty$, after rescaling $\mathscr{A}$ and $C(\cdot)$ by $\alpha$. Evidently the analogue of Ripa's theorem is lost, while the constrained version of $\mathscr{A}$ takes the form

$$
\begin{aligned}
\mathscr{A}= & \int\left\{-\frac{g}{2 f^{2}} \frac{\mathrm{d} f}{\mathrm{~d} y}\left(h^{\prime}\right)^{2}+\left(H+h^{\prime}\right) \int_{0}^{q^{\prime}}\left[C^{\prime}(Q+\xi)-C^{\prime}(Q)\right] \mathrm{d} \xi\right\} \mathrm{d} \boldsymbol{x} \\
& -\sum_{i} \oint_{\partial D_{i}} \frac{g}{2 f} \hat{\boldsymbol{n}} \cdot \hat{\boldsymbol{y}}\left(h^{\prime}\right)^{2} \mathrm{~d} s,
\end{aligned}
$$

where $C(\cdot)$ is given by (4.2) with $K \equiv 0$ and $\alpha=1$. The lateral boundary contributions to the pseudomomentum (4.8), which are not present in QG dynamics (Shepherd 1989), are consistent with what is found in SG dynamics (Kushner \& Shepherd $1995 a$ ). Since we have assumed a zonally symmetric domain, any boundary must be a wall at constant $y$, and there are only four choices: (i) no boundaries; (ii) northern boundary only, $y \leqslant y_{2}$; (iii) southern boundary only, $y_{1} \leqslant y$; or (iv) zonal channel, $y_{1} \leqslant y \leqslant y_{2}$. A northern boundary will always give a negative contribution to $\mathscr{A}$, while a southern boundary will always give a positive contribution; this agrees with the SG case provided one restricts attention to small Rossby number.

With no boundaries, $\mathscr{A}$ is sign-definite if $Q_{y}>0$ in the variable- $f$ case, or if $Q_{y} \neq 0$ in the $f$-plane case. The latter case is analogous to the QG Charney-Stern theorem (Shepherd 1989). With a northern boundary only, $\mathscr{A}$ is sign-definite if $Q_{y}>0$ in both the variable- $f$ and $f$-plane cases. With a southern boundary only, $\mathscr{A}$ is sign-definite if $Q_{y}<0$ in the $f$-plane case. Finally, in the case of a zonal channel with two boundaries, no stability result is forthcoming. In all the above stability conditions, the $f$-plane cases are the same as in the SG model (Kushner \& Shepherd 1995a).

\subsection{Axisymmetric basic flows}

For completeness, we consider the case where the basic flow (and domain geometry) is axisymmetric; this requires $f(y)=$ const. Because the basic flow has curvature, its ageostrophic component must be non-zero (except at lateral boundaries); the basicflow curvature is weak in the relevant sense, and thus consistent with the scaling assumptions of Salmon's model, provided the Rossby number is small. The analysis proceeds exactly as in $\S 4.1$, with the substitutions

$$
\mathscr{M} \rightarrow \mathscr{J}, \quad G(\cdot) \rightarrow L(\cdot) \quad \text { with } \quad L(Q(r))=W_{G}(r)+\frac{1}{2} f r^{2},
$$


where $W_{G} \equiv \hat{\boldsymbol{z}} \cdot\left(\boldsymbol{x} \times \boldsymbol{U}_{G}\right)=(r g / f) \mathrm{d} N / \mathrm{d} r$. This leads to the finite-amplitude waveactivity invariant

$\mathscr{A}=\int\left\{\frac{1}{2}\left(H+h^{\prime}\right)\left|\boldsymbol{u}_{G}^{\prime}\right|^{2}+h^{\prime}\left(\frac{W_{G}}{r^{2}}+\alpha\right) w_{G}^{\prime}+\frac{1}{2} g\left(h^{\prime}\right)^{2}+\left(H+h^{\prime}\right) \int_{0}^{q^{\prime}}\left[C^{\prime}(Q+\xi)-C^{\prime}(Q)\right] \mathrm{d} \xi\right\} \mathrm{d} \boldsymbol{x}$.

In this case the analogue of Ripa's small-amplitude stability theorem is that the basic flow is stable if a value of $\alpha$ exists such that

$$
\left(\frac{W_{G}}{r}+\alpha r\right)^{2}<g H
$$

and

$$
C^{\prime \prime}(Q)=\frac{\Psi_{r}+\alpha r H}{Q_{r}}>0 .
$$

On the other hand, after the geostrophic constraint is imposed $\mathscr{A}$ takes the form

$$
\begin{aligned}
\mathscr{A} & =\int\left\{\frac{g^{2}}{2 f^{2}}\left(H+h^{\prime}\right)\left|\nabla h^{\prime}\right|^{2}+\frac{1}{2}\left[g-\frac{1}{r} \frac{\mathrm{d}}{\mathrm{d} r}\left(r \frac{g^{2}}{f^{2}} \frac{\mathrm{d} N}{\mathrm{~d} r}\right)-2 \alpha \frac{g}{f}\right]\left(h^{\prime}\right)^{2}\right. \\
& \left.+\left(H+h^{\prime}\right) \int_{0}^{q^{\prime}}\left[C^{\prime}(Q+\xi)-C^{\prime}(Q)\right] \mathrm{d} \xi\right\} \mathrm{d} \boldsymbol{x} \\
& +\sum_{i} \oint_{\partial D_{i}} \frac{g}{2 f}\left(\frac{W_{G}}{r}+\alpha r\right) \hat{\boldsymbol{n}} \cdot \hat{\boldsymbol{r}}\left(h^{\prime}\right)^{2} \mathrm{~d} s .
\end{aligned}
$$

Evidently $\mathscr{A}$ is positive definite and convex, and nonlinear stability is provable, whenever there exists a value of $\alpha$ and constants $c_{1}, c_{2}, k_{1}, k_{2}, b_{1}^{(i)}$ and $b_{2}^{(i)}$ such that

$$
\begin{gathered}
0<c_{1} \leqslant C^{\prime \prime}(Q)=\frac{\Psi_{r}+\alpha r H}{Q_{r}} \leqslant c_{2}<\infty, \\
0<k_{1} \leqslant f-\zeta_{G}-2 \alpha=f-\frac{1}{r} \frac{\mathrm{d}}{\mathrm{d} r}\left(W_{G}+\alpha r^{2}\right) \leqslant k_{2}<\infty, \\
0<b_{1}^{(i)} \leqslant\left(\frac{W_{G}}{r}+\alpha r\right) \hat{\boldsymbol{n}} \cdot \hat{\boldsymbol{r}} \leqslant b_{2}^{(i)}<\infty \text { on } \partial D_{i} .
\end{gathered}
$$

There is no need to discuss these conditions in any detail, except in the special case of an unbounded domain where certain differences with the zonally symmetric case emerge. In that case, taking $r \rightarrow \infty$ in $(4.11 a)$ requires $\alpha=0$. But then $(4.11 b)$ requires $\Psi_{r} / Q_{r}>0$, which will be difficult to satisfy. (Nore \& Shepherd 1997, Appendix A, show that the corresponding condition for the shallow-water system can never be satisfied for a localized vortex; the same result holds for QG dynamics.) On the other hand, in an unbounded domain the boundary contributions to (4.12) disappear, and condition $(4.13 c)$ drops out, provided $W_{G}$ is finite at $r=0, W_{G}\left(h^{\prime}\right)^{2} \rightarrow 0$ as $r \rightarrow \infty$, and $r^{2}\left(h^{\prime}\right)^{2} \rightarrow 0$ as $r \rightarrow \infty$. The last condition is required in any case for finite disturbance energy. Then the stability conditions (4.13) are essentially analogous to those of QG dynamics, and one is free to take $\alpha \neq 0$. If the energy is not used in the construction of $\mathscr{A}$ (in which case the wave-activity invariant is just the angular pseudomomentum), then the resulting stability condition is $Q_{r}<0$. This differs from the QG axisymmetric Charney-Stern theorem, which requires only that $Q_{r} \neq 0$; in Salmon's model, only cyclonic vortices appear to be provably stable using the angular pseudomomentum alone. 


\section{Summary and discussion}

The symmetry-based conservation laws of Salmon's (1983) model of nearly geostrophic shallow-water dynamics have been used to derive exact nonlinear waveactivity invariants and stability theorems for disturbances to steady flows. It is shown that steady solutions of Salmon's model must have a non-vanishing ageostrophic flow component unless the flow is straight. The extremal condition $\delta \mathscr{A}=0$ is shown to imply a boundary condition for this ageostrophic flow component, as well as an ageostrophic correction to the Bernoulli function, which are identical to those derived by Salmon (1983 and unpublished notes) and Allen \& Holm (1996) directly from Hamilton's principle. This boundary condition (2.10) is shown to be accurate for weakly curved boundaries. The presence of lateral boundaries produces boundary contributions to the wave-activity invariants, once the geostrophic constraint is incorporated, and these boundary terms affect the stability criteria. It must be emphasized that these boundary terms represent real physical effects associated with the existence of coastal Kelvin waves in both the SG model and Salmon's model (Allen et al. 1990a; Kushner et al. 1997). Such boundary terms do not arise in the QG model, which does not support coastal Kelvin waves.

Salmon's model (in the shallow-water context) is found to possess two distinct kinds of stability theorems: an analogue of Ripa's (1983) small-amplitude stability theorem for shallow-water flow, and finite-amplitude analogues of Kushner \& Shepherd's $(1995 a, b)$ SG stability theorems. That the two kinds of theorems have complementary stability conditions is demonstrated by an explicit example. In both cases, the stability conditions consist of Arnol'd's (1966) condition $\nabla \Psi / \nabla Q>0$ plus an additional condition that serves to limit the interactions between coastal Kelvin waves and the rest of the dynamics. In the first case, the additional condition is that the Froude number be less than unity; and in the second case, that the flow be cyclonic along lateral boundaries (there is also one other condition that is satisfied if the Rossby number is less than unity). Physically, either condition ensures that the coastal Kelvin waves in Salmon's model - which propagate cyclonically at phase speeds greater than $(g H)^{1 / 2}$ - cannot be Doppler-shifted into phase-locked interactions with the low-frequency interior dynamics, or with a coastal Kelvin wave on another boundary. No such additional stability condition is required in QG dynamics.

A significant technical difference with the SG analysis of Kushner \& Shepherd $(1995 a, b)$ is that the latter found it necessary to work in transformed isentropicgeostrophic coordinates, in order to deal with the potential-vorticity invertibility principle of SG dynamics; this enormously complicated the treatment of the boundary dynamics, and led to a number of unsatisfactory restrictions on the results particularly at finite amplitude. These technical difficulties would be expected to arise with any model formulated in transformed coordinates, including Salmon's (1985) generalized SG model. The present analysis, in contrast, has been performed entirely in physical coordinates, and the boundary contributions are therefore easy to handle. In particular, it is clearly seen how the geostrophic constraint produces lateral boundary contributions to wave-activity invariants.

Detailed comparison of analytical and numerical solutions of approximate shallowwater models (Allen et al. 1990a, b; Barth, Allen \& Newberger 1990) has shown that Salmon's (1983) model is not as accurate as other balanced models that do not possess symmetry-based conservation laws. However, Salmon's model represents an important prototype for potentially more accurate constrained Hamiltonian balanced models, as has recently become evident from the work of Allen \& Holm (1996) and 
McIntyre \& Roulstone (1997). Those studies show that constraining the velocity to the mass field within a Hamiltonian context leads to a balanced shallow-water model having the form

$$
\begin{gathered}
\frac{\partial \boldsymbol{u}_{C}}{\partial t}+q h \hat{\boldsymbol{z}} \times \boldsymbol{u}=-\nabla b, \\
\frac{\partial h}{\partial t}+\nabla \cdot(h \boldsymbol{u})=0,
\end{gathered}
$$

where $\boldsymbol{u}_{C}$ is the constraint velocity, which appears in all conservation laws (2.3)-(2.7), $\boldsymbol{u}$ is the particle (i.e. advecting) velocity, $b$ is the appropriate generalized Bernoulli function, and $q \equiv\left[f+\hat{z} \cdot\left(\nabla \times \boldsymbol{u}_{C}\right)\right] / h$ is the potential vorticity associated with the constraint velocity. Salmon's (1983) model is just the special case $\boldsymbol{u}_{C}=\boldsymbol{u}_{G}$. It is easy to see that $(5.1 a, b)$ imply $q_{t}+\boldsymbol{u} \cdot \nabla q=0$. Steady solutions of such a model will clearly satisfy the Bernoulli relationship (3.1), and a pseudoenergy invariant (3.3) for disturbances to a steady basic flow will therefore be constructable as in $\S 3$ with the relations (3.4)-(3.6). The properties (3.7) will again hold, and it is easy to verify that the extremal condition will then reduce to

$$
\delta \mathscr{A}=\int\left\{-B^{\prime} \delta h-\hat{z} \cdot\left(\nabla \Psi^{\prime} \times \delta \boldsymbol{u}_{C}\right)\right\} \mathrm{d} \boldsymbol{x},
$$

where $B^{\prime}$ is the difference between $B$ and the usual Bernoulli function evaluated using the constraint velocity, i.e. $B^{\prime} \equiv B-g N-\left|\boldsymbol{U}_{C}\right|^{2} / 2$, and $\Psi^{\prime}$ is defined by $H\left(\boldsymbol{U}-\boldsymbol{U}_{C}\right)=\hat{z} \times \nabla \Psi^{\prime}$. The expression (5.2) is the generalization of (3.8a), and reflects the velocity-split nature of this class of models. The term involving $\delta \boldsymbol{u}_{C}$ will then be expressible in terms of some differential operator on $\delta h$, whose inversion will yield a boundary contribution to $\delta \mathscr{A}$. Thus the extremal condition $\delta \mathscr{A}=0$ will produce a boundary condition for $\left(\boldsymbol{U}-\boldsymbol{U}_{C}\right)$ as well as an expression for $B^{\prime}$, which are the generalizations of (2.10) and (3.2a). The expression (3.9) for the pseudoenergy will then apply, with $\boldsymbol{U}_{G}$ and $\boldsymbol{u}_{G}^{\prime}$ replaced by $\boldsymbol{U}_{C}$ and $\boldsymbol{u}_{C}^{\prime}$, respectively, and an analogue of Ripa's theorem corresponding to (3.12) will follow. Whether a nonlinear stability theorem analogous to (3.14) can be obtained after expressing $\boldsymbol{u}_{C}^{\prime}$ in terms of $h^{\prime}$ will, of course, depend on the particular form of the constraint. In a completely analogous way, the expressions (4.4) and (4.10), and the small-amplitude stability conditions (4.5) and (4.11), will also apply to such general constrained Hamiltonian balanced models, with the obvious substitutions.

The fact that the class of general constrained Hamiltonian balanced models discussed above possesses analogues of Ripa's theorem suggests that the coastal Kelvin waves in such models must have phase speeds $c$ greater than or equal to $(g H)^{1 / 2}$, in the light of the subsonic condition (3.12a). This is intriguing, because Salmon's model stands out as the only balanced model considered by Allen et al. (1990a) with $c \geqslant(g H)^{1 / 2}$; all the others (with the exception of the QG model, which filters the coastal Kelvin waves) have $c<(g H)^{1 / 2}$. (In all cases, including Salmon's model, $c \rightarrow(g H)^{1 / 2}$ in the low-frequency, long-wave limit.) Of the latter models, only the SG model is Hamiltonian, and as noted by McIntyre \& Roulstone (1997) the SG model does not fit into the above framework. Allen \& Holm (1996) show that the SG model can indeed be cast in the form (5.1) with a certain choice of $\boldsymbol{u}_{C}$, but at the price of changing the natural definition of $\mathscr{H}: \boldsymbol{u}$ in (2.3) is replaced by $\boldsymbol{u}_{G}$, not by $\boldsymbol{u}_{C}$. Thus there is no contradiction between our general results and the known properties of the SG model. (In particular, the SG model does not appear to possess an analogue of Ripa's theorem.) 
In order to better understand this dichotomy, we briefly review the nature of the dispersion relation for coastal Kelvin waves. Allen et al. $(1990 a, \S 4 b)$ show that the coastal Kelvin waves in all the balanced models they consider, except Salmon's model, have phase speeds given by

$$
c=\frac{f}{\ell},
$$

where $\ell$ is the inverse of the e-folding (trapping) scale, which is in accordance with the shallow-water equations. For the shallow-water equations, $\ell=f /(g H)^{1 / 2}$ so that $c=(g H)^{1 / 2}$, where $H$ is the (uniform) depth of the undisturbed fluid. The balanced models, however, introduce the distortion

$$
\ell=\frac{f}{(g H)^{1 / 2}}\left(1+\frac{g H k^{2}}{f^{2}}\right)^{1 / 2},
$$

where $k$ is the along-coast wavenumber, and this has the effect of slowing down the coastal Kelvin waves in the high-frequency large- $k$ limit. Salmon's model contains the distortion (5.4), but also introduces a further distortion in that (5.3) is replaced by

$$
c=\frac{g H \ell}{f} .
$$

The distortion (5.5) is a consequence of the ageostrophic contribution to the Bernoulli function (3.2a), and has the effect of speeding up the coastal Kelvin waves in the high-frequency large- $k$ limit.

It is easy to show that the coastal Kelvin waves in the general constrained Hamiltonian balanced models discussed above must, as with Salmon's model, have phase speeds greater than or equal to $\left(g h^{1 / 2}\right)$. For such models, the small-amplitude pseudoenergy $\mathscr{E}$ and pseudomomentum $\mathscr{P}$ associated with zero- $q^{\prime}$ disturbances to a resting basic state reduce to

$$
\mathscr{E}=\int\left\{\frac{1}{2} H\left|\boldsymbol{u}_{C}^{\prime}\right|^{2}+\frac{1}{2} g\left(h^{\prime}\right)^{2}\right\} \mathrm{d} \boldsymbol{x}, \quad \mathscr{P}=\int h^{\prime} u_{C}^{\prime} \mathrm{d} \boldsymbol{x} .
$$

The phase speed $c$ of a steadily propagating distrubance is give by $c=\mathscr{E} / \mathscr{P}$ (e.g. Shepherd 1994), and by completing the square it is easy to see from (5.6) that $\mathscr{E} \geqslant(g h)^{1 / 2}|\mathscr{P}|$.

The different nature of the large- $k$ distortion of the coastal Kelvin waves in the two kinds of balanced models has certain implications. Allen et al. (1990) argue that the 'supersonic' distortion of Salmon's model is unattractive because it could lead to numerical problems. Such problems could, of course, be overcome with a semi-implicit time-stepping scheme. On the other hand, it could be argued that the 'supersonic' distortion has the merit of reducing the strength of the interactions between the large- $k$ coastal Kelvin waves - which are in any case not well represented in any balanced model - and the rest of the balanced dynamics. Such interactions are, in contrast, potentially allowed in the other balanced models which contain a 'subsonic' distortion, and could lead to physically spurious results. In particular, flows that were provably stable in shallow-water dynamics according to Ripa's theorem might be unstable in such models. This is an interesting prospect for further investigation.

Balanced models are intended to be reduced descriptions of an underlying 'primitive' dynamics. It is therefore arguable that any flow that is stable under the primitive dynamics ought also to be stable under any physically reasonable balanced dynamics. (Though not vice versa.) The fact that Salmon's model contains an analogue of Ripa's shallow-water stability theorem is, therefore, a point very much in its favour. 
This paper is based on research partially carried out at the Isaac Newton Institute for Mathematical Sciences, Cambridge, UK during the programme 'Mathematics of Atmosphere and Ocean Dynamics', July-December 1996. The research has also been supported by grants from the Natural Sciences and Engineering Research Council and the Atmospheric Environment Service of Canada. The authors are grateful to Dr. J. Vanneste for a helpful suggestion. Newton Institute publication NI197011-AOD.

\section{Appendix A. Equations for $\boldsymbol{u}_{A G}$}

Differentiating (2.1b) with respect to either $x$ or $y$ and using (2.8) yields evolution equations for the two components of geostrophic velocity. Consistency between those evolution equations and (2.9) then yields an implicit determination of $\boldsymbol{u}_{A G}$ via the two equations

$$
\begin{aligned}
& \left\{\frac{g}{f} \nabla^{2}-q\right\}\left(h v_{A G}\right)+\frac{g}{f^{2}} \frac{\mathrm{d} f}{\mathrm{~d} y} \frac{\partial}{\partial x}\left(h u_{A G}\right)=-\frac{g}{f} \frac{\partial^{2}\left(h u_{G}\right)}{\partial x \partial y}-\frac{g}{f} \frac{\partial^{2}\left(h v_{G}\right)}{\partial y^{2}}+h q v_{G}-\frac{\partial B_{G}}{\partial x}, \\
& \left\{\nabla^{2}-\frac{f q}{g}\right\}\left(\frac{g h}{f} u_{A G}\right)+\frac{g}{f^{2}} \frac{\mathrm{d} f}{\mathrm{~d} y} \frac{\partial}{\partial x}\left(h v_{A G}\right)=-\frac{g}{f} \frac{\partial^{2}\left(h v_{G}\right)}{\partial x \partial y}-\frac{g}{f} \frac{\partial^{2}\left(h u_{G}\right)}{\partial x^{2}}+h q u_{G}+\frac{\partial B_{G}}{\partial y},
\end{aligned}
$$

where $B_{G}$ is the geostrophic Bernoulli function. For given $h$, (A 1)-(A 2) represents a coupled set of linear elliptic second-order differential equations for $u_{A G}$ and $v_{A G}$. The equations have a unique solution for $u_{A G}$ and $v_{A G}$ for given boundary values in a closed domain provided $q>0$ (Courant \& Hilbert 1962, §IV.7.2). The condition $q>0$ is guaranteed for small Rossby number, and will hold for all time if it holds initially. The two boundary conditions (2.2) and (2.10) determine two orthogonal components of $\boldsymbol{u}_{A G}$ (for given $h$ ), and therefore provide the needed boundary values for $u_{A G}$ and $v_{A G}$. A unique solution also exists if the domain is periodic in one or both directions. For unbounded domains, on the other hand, it is difficult to make definite statements about the solution properties of (A 1)-(A 2).

\section{Appendix B. Variational relationships}

The following variational relationships are useful for verifying the analysis of $\S \S 3-4$ :

$$
\begin{gathered}
\delta \boldsymbol{u}_{G}=\frac{g}{f} \hat{z} \times \nabla \delta h, \quad \delta \eta=\delta h, \\
h \delta q=\hat{z} \cdot\left(\nabla \times \delta \boldsymbol{u}_{G}\right)-q \delta h=\nabla \cdot\left(\frac{g}{f} \nabla \delta h\right)-q \delta h, \\
\delta \mathscr{H}=\int\left\{h \boldsymbol{u}_{G} \cdot \delta \boldsymbol{u}_{G}+\left(\frac{1}{2}\left|\boldsymbol{u}_{G}\right|^{2}+g \eta\right) \delta \eta\right\} \mathrm{d} \boldsymbol{x} \\
=\int\left\{\frac{g^{2}}{2 f^{2}}|\nabla \eta|^{2}+g \eta-\nabla \cdot\left(\frac{g^{2} h}{f^{2}} \nabla \eta\right)\right\} \delta h \mathrm{~d} \boldsymbol{x}+\sum_{i} \oint_{\partial D_{i}} \frac{g^{2} h}{f^{2}}(\nabla \eta \cdot \hat{\boldsymbol{n}}) \delta h \mathrm{~d} s,
\end{gathered}
$$

where $d s$ is arclength along $\partial D_{i}$,

$$
\begin{aligned}
\delta \mathscr{C} & =\int\left\{C(q) \delta h+h C^{\prime}(q) \delta q\right\} \mathrm{d} \boldsymbol{x} \\
& =\int\left\{\left[C(q)-q C^{\prime}(q)\right] \delta h-\hat{z} \cdot\left(\nabla C^{\prime}(q) \times \delta \boldsymbol{u}_{G}\right)\right\} \mathrm{d} \boldsymbol{x}+\sum_{i} \oint_{\partial D_{i}} C^{\prime}(q) \delta \boldsymbol{u}_{G} \cdot \mathrm{d} \ell
\end{aligned}
$$




$$
\begin{gathered}
=\int\left\{C(q)-q C^{\prime}(q)+\nabla \cdot\left(\frac{g}{f} \nabla C^{\prime}(q)\right)\right\} \delta h \mathrm{~d} \boldsymbol{x} \\
+\sum_{i} \oint_{\partial D_{i}} \frac{g}{f}\left[C^{\prime}(q) \nabla \delta h \cdot \hat{\boldsymbol{n}}-\left(\nabla C^{\prime}(q) \cdot \hat{\boldsymbol{n}}\right) \delta h\right] \mathrm{d} s, \\
\delta \Gamma_{i}=\oint_{\partial D_{i}} \delta \boldsymbol{u}_{G} \cdot \mathrm{d} \ell=\oint_{\partial D_{i}} \frac{g}{f}(\nabla \delta h \cdot \hat{\boldsymbol{n}}) \mathrm{d} s, \\
\delta \mathscr{M}=\int\left\{\left(u_{G}-F\right) \delta h+h \delta u_{G}\right\} \mathrm{d} \boldsymbol{x}=\int\left\{u_{G}-F+\frac{\partial}{\partial y}\left(\frac{g h}{f}\right)\right\} \delta h \mathrm{~d} \boldsymbol{x}-\sum_{i} \oint_{\partial D_{i}} \frac{g h}{f} \delta h \mathrm{~d} s, \\
\delta \mathscr{J}=\int\left\{\left(w_{G}+\frac{1}{2} f r^{2}\right) \delta h+h \delta w_{G}\right\} \mathrm{d} \boldsymbol{x} \\
=\int\left\{w_{G}+\frac{1}{2} f r^{2}-\frac{1}{r} \frac{\partial}{\partial r}\left(\frac{r^{2} g h}{f}\right)\right\} \delta h \mathrm{~d} \boldsymbol{x}+\sum_{i} \oint_{\partial D_{i}} \frac{r g h}{f} \delta h \mathrm{~d} s,
\end{gathered}
$$

where $w_{G} \equiv \hat{z} \cdot\left(\boldsymbol{x} \times \boldsymbol{u}_{G}\right)=(r g / f) \partial \eta / \partial r$.

\section{REFERENCES}

Allen, J. S., Barth, J. A. \& Newberger, P. A. 1990a On intermediate models for barotropic continental shelf and slope flow fields. Part I: Formulation and comparison of exact solutions. J. Phys. Oceanogr. 20, 1017-1042.

Allen, J. S., Barth, J. A. \& Newberger, P. A. $1990 b$ On intermediate models for barotropic continental shelf and slope flow fields. Part III: Comparison of numerical model solutions in periodic channels. J. Phys. Oceanogr. 20, 1949-1973.

Allen, J. S. \& Holm, D. D. 1996 Extended-geostrophic Hamiltonian models for rotating shallow water motion. Physica D 98, 229-248.

ARNOL'D, V. I. 1966 On an a priori estimate in the theory of hydrodynamical stability. Izv. Vyssh. Uchebn. Zaved. Matematika 54(5), 3-5. (English transl. Am. Math. Soc. Transl., Series 2 79, 267-269 (1969).)

Barth, J. A., Allen, J. S. \& Newberger, P. A. 1990 On intermediate models for barotropic continental shelf and slope flow fields. Part II: Comparison of numerical model solutions in doubly periodic domains. J. Phys. Oceanogr. 20, 1044-1076.

Boкhove, O. 1996 On balanced models in geophysical fluid dynamics. PhD thesis, Department of Physics, University of Toronto.

Courant, R. \& Hilbert, D. 1962 Methods of Mathematical Physics, Volume II. John Wiley \& Sons.

FORD, R. 1993 Gravity wave generation by vortical flows in a rotating frame. PhD thesis, Department of Applied Mathematics and Theoretical Physics, Cambridge University.

Holm, D. D., Marsden, J. E., Ratiu, T. \& Weinstein, A. 1985 Nonlinear stability of fluid and plasma equilibria. Phys. Rep. 123, 1-116.

Hoskins, B. J. 1975 The geostrophic momentum approximation and the semi-geostrophic equations. J. Atmos. Sci. 32, 233-242.

Kushner, P. J., McIntyre, M. E. \& Shepherd, T. G. 1997 Coupled Kelvin-wave and mirage-wave instabilities in semi-geostrophic dynamics. J. Phys. Oceanogr. in press.

Kushner, P. J. \& Shepherd, T. G. $1995 a$ Wave-activity conservation laws and stability theorems for semi-geostrophic dynamics. Part 1. Pseudomomentum-based theory. J. Fluid Mech. 290, 67-104.

Kushner, P. J. \& ShePherd, T. G. $1995 b$ Wave-activity conservation laws and stability theorems for semi-geostrophic dynamics. Part 2. Pseudoenergy-based theory. J. Fluid Mech. 290, 105-129.

LoRenZ, E. N. 1960 Energy and numerical weather prediction. Tellus 12, 364-373.

Magnusdottir, G. \& Schubert, W. H. 1990 The generalization of semi-geostrophic theory to the $\beta$-plane. J. Atmos. Sci. 47, 1714-1720. 
MagnusdottiR, G. \& Schubert, W. H. 1991 Semigeostrophic theory on the hemisphere. J. Atmos. Sci. 48, 1449-1456.

McIntyre, M. E. \& Roulstone, I. 1997 Hamiltonian balanced models: constraints, slow manifolds and velocity splitting. J. Fluid Mech., submitted.

McIntyre, M. E. \& Shepherd, T. G. 1987 An exact local conservation theorem for finite-amplitude disturbances to non-parallel shear flows, with remarks on Hamiltonian structure and on Arnol'd's stability theorems. J. Fluid Mech. 181, 527-565.

Nore, C. \& Shepherd, T. G. 1997 A Hamiltonian weak-wave model for shallow-water flow. Proc. R. Soc. Lond. A 453, 563-580.

Pedlosky, J. 1987 Geophysical Fluid Dynamics, 2nd edn. Springer.

RIPA, P. 1983 General stability conditions for zonal flows in a one-layer model on the $\beta$-plane or the sphere. J. Fluid Mech. 126, 463-489.

RIPA, P. 1991 General stability conditions for a multi-layer model. J. Fluid Mech. 222, 119-137.

SAdourny, R. 1975 The dynamics of finite-difference models of the shallow water equations. $J$. Atmos. Sci. 32, 680-689.

Salmon, R. 1983 Practical use of Hamilton's principle. J. Fluid Mech. 132, 431-444.

SALmon, R. 1985 New equations for nearly geostrophic flow. J. Fluid Mech. 153, 461-477.

SHEPHERD, T. G. 1989 Nonlinear saturation of baroclinic instability. Part II: Continuously stratified fluid. J. Atmos. Sci. 46, 888-907.

SHEPHERD, T. G. 1990 Symmetries, conservation laws, and Hamiltonian structure in geophysical fluid dynamics. Adv. Geophys. 32, 287-338.

SHEPHERD, T. G. 1992 Arnol'd stability applied to fluid flow: successes and failures. In Nonlinear Phenomena in Atmospheric and Oceanic Sciences (ed. G. F. Carnevale \& R. T. Pierrehumbert), pp. 187-206. Springer.

ShePherd, T. G. 1993 A unified theory of available potential energy. Atmos.-Ocean 31, 1-26.

ShePherd, T. G. 1994 Applications of Hamiltonian theory to geophysical fluid dynamics. In Geometrical Methods in Fluid Dynamics (ed. R. Salmon \& B. Ewing-Deremer). Woods Hole Oceanographic Institution Tech. Rep. WHOI-94-12, pp. 113-152.

ShutTs, G. J. 1989 Planetary semi-geostrophic equations derived from Hamilton's principle. J. Fluid Mech. 208, 545-573.

Warn, T., Bokhove, O., Shepherd, T. G. \& Vallis, G. K. 1995 Rossby-number expansions, slaving principles, and balance dynamics. Q. J. R. Met. Soc. 121, 723-739. 